\title{
A note on monitoring time-varying parameters in an autoregression
}

\author{
Frédéric Carsoule ${ }^{1}$, Philip Hans Franses ${ }^{2 *}$ \\ ${ }^{1}$ Tinbergen Institute, Erasmus University Rotterdam, Ondlaan 50, 3000 DR Rotterdam, The \\ Netherlands \\ ${ }^{2}$ Econometric Institute, office H11-34, Erasmus University Rotterdam, P.O. Box 1738, \\ NL-3000 DR Rotterdam, The Netherlands (e-mail: franses@few.eur.nl)
}

\begin{abstract}
We develop a sequential testing approach for a structural change in the parameters of an autoregression, which amounts to an adaptation of the monitoring procedure, outlined in Chu, Stichcombe and White (1996). This procedure has a controlled asymptotic size as one repeats the test. Our method can be used as a general misspecification test. We apply our method to monthly US industrial production in order to investigate if its autoregressive behavior and/or its innovation variance have changed during the twentieth century.
\end{abstract}

Key words: Structural change; autoregression; misspecification test

\section{Introduction}

It is important to examine parameter stability in autoregressive time series models. For example, when shifts in the autoregressive parameters are neglected, one generates biased out-of-sample forecasts. Additionally, when such shifts in the variance are overlooked, the prediction intervals will be too wide or too narrow. Also from a descriptive point of view, it is interesting to examine if, for example, cyclical patterns (as indicated by the size of the complex roots of the AR polynomial) or persistence properties have changed over time. In this paper, we therefore develop a sequential testing approach for structural change in the autoregressive and variance parameters of an autoregression, which can be implemented as a general misspecification test. In order to obtain a method with a controlled asymptotic size as we repeat the test, we adapt the monitoring procedure as it was proposed in Chu, Stinchcombe and White (1996). We apply our method to monthly US industrial production in order to investigate if its

\footnotetext{
* We thank an anonymous referee for several helpful comments.
} 
autoregressive behavior and/or its innovation variance have changed during the twentieth century.

The outline of our paper is as follows. In section 2, we present the model and the relevant test statistic. In section 3 , we outline the monitoring procedure, and we evaluate it using simulation experiments. In section 4 , we report on our empirical application. In section 5, we conclude with some remarks.

\section{The model and the test statistics}

In this section we first consider a general autoregression and next we illustrate matters for a second order model, as this last model will be used in our empirical illustration.

\subsection{Autoregression of order $p$}

Consider a time series $\left\{y_{t}\right\}$, which can be described by an autoregressive process of order $p[\operatorname{AR}(p)]$, that is by

$$
y_{t}=\phi_{1, t} y_{t-1}+\cdots+\phi_{p, t} y_{t-p}+\varepsilon_{t}
$$

with $\varepsilon_{t} \mid \mathscr{F}_{t-1} \sim \mathcal{N}\left(0, \sigma_{t}^{2}\right)$, and where $\mathscr{F}_{t-1}$ is the $\sigma$-field generated by $y_{1-p}, \ldots$, $y_{0}, \varepsilon_{1}, \ldots, \varepsilon_{t-1}$.

We are interested in examining if the parameters of the autoregressive model are stable, that is, if the parameters $\phi_{t}=\left(\phi_{1, t}, \ldots, \phi_{p, t}\right)^{\prime}$ and $\sigma_{t}^{2}$ are constant over time. More precise, it is assumed first that the model is constant up to time $m: \sigma_{1}^{2}=\cdots=\sigma_{m}^{2}=\sigma_{0}^{2}, \phi_{1}=\cdots=\phi_{m}=\phi$, and we subsequently consider the null hypothesis

$$
H_{0}:\left(\phi_{t}^{\prime}, \sigma_{t}^{2}\right)=\left(\phi^{\prime}, \sigma_{0}^{2}\right), \quad t=m+1, \ldots
$$

against the alternative hypothesis

$$
H_{1}:\left(\phi_{t}^{\prime}, \sigma_{t}^{2}\right) \text { changes at some } t \geq m+1 \text {. }
$$

Furthermore, if $H_{0}$ gets rejected, we want to have some indication as to which parameters are subject to change. For example it can be the variance of $\varepsilon_{t}$, the AR parameters, or both. Therefore, we will rely on the score process, that is, the vector of partial derivatives of the log-likelihood. Under the null hypothesis, the time-varying parameter model (1) reduces to

$$
y_{t}=\phi_{1} y_{t-1}+\cdots+\phi_{p} y_{t-p}+\varepsilon_{t}
$$

where we assume that the zeros of the polynomial $1-\phi_{1} z-\cdots-\phi_{p} z^{p}$ lie outside the unit circle and $\varepsilon_{t} \mid \mathscr{F}_{t-1} \sim \mathscr{N}\left(0, \sigma_{0}^{2}\right)$. Hence we assume covariancestationarity.

Denote by $\theta$ the $p+1$ vector of unknown parameters, that is $\theta=\left(\phi^{\prime}, \sigma^{2}\right)^{\prime}$, and denote by $l_{m}(\theta)$ the conditional log-likelihood at time $m$, that is, 


$$
l_{m}(\theta)=\sum_{t=1}^{m} \log f\left(y_{t} \mid \mathscr{F}_{t-1}, \theta\right)
$$

where

$$
\log f\left(y_{t} \mid \mathscr{F}_{t-1}, \theta\right)=-1 / 2 \log 2 \pi-1 / 2 \log \sigma^{2}-\left(1 / 2 \sigma^{2}\right) \varepsilon_{t}^{2} .
$$

Furthermore, use $\rho_{t}(\theta)$ to denote the conditional score function at time $t$, that is,

$$
\rho_{t}(\theta)=\nabla \log f\left(y_{t} \mid \mathscr{F}_{t-1}, \theta\right),
$$

where

$$
\nabla \log f\left(y_{t} \mid \mathscr{F}_{t-1}, \theta\right)=\left(\frac{\partial \log f\left(y_{t} \mid \mathscr{F}_{t-1}, \theta\right)}{\partial \theta_{j}}\right)_{j=1,2, \ldots, p+1}
$$

is the gradient vector of $\log f\left(y_{t} \mid \mathscr{F}_{t-1}, \theta\right)$.

From the expression (6) of the log-likelihood, it is easy to see that the components of the score function at time $t$ are given by

$$
\left\{\begin{array}{l}
\frac{\partial \log f\left(y_{t} \mid \mathscr{F}_{t-1}, \theta\right)}{\partial \phi_{j}}=y_{t-j} \frac{\varepsilon_{t}}{\sigma^{2}} \quad j=1, \ldots, p \\
\frac{\partial \log f\left(y_{t} \mid \mathscr{F}_{t-1}, \theta\right)}{\partial \sigma^{2}}=\frac{1}{2 \sigma^{2}}\left(\frac{\varepsilon_{t}^{2}}{\sigma^{2}}-1\right) .
\end{array}\right.
$$

For further reference, denote by $\Psi_{\lambda}^{m}(\theta)$ the score process, that is,

$$
\Psi_{\lambda}^{m}(\theta)=m^{-1 / 2} \sum_{t=1}^{[m \lambda]} \rho_{t}(\theta)
$$

for $\lambda \in R_{+}$. Our test below will be based on this score process.

Let $I(\theta)$ be the information matrix defined by

$$
I(\theta)=-E\left(\frac{\partial^{2} \log f\left(y_{t} \mid \mathscr{\mathscr { F }}_{t-1}, \theta\right)}{\partial \theta_{j} \partial \theta_{k}}\right)_{j, k=1, \ldots, p+1}
$$

The autocovariance function of the stationary process $y_{t}$ in (4) is defined by

$$
\gamma(h)=\operatorname{cov}\left(y_{t+h}, y_{t}\right) .
$$

Given this function, the information matrix is defined by 


$$
I(\theta)=\frac{1}{\sigma^{2}}\left(\begin{array}{cccc}
\gamma(0) & \cdots & \gamma(p-1) & 0 \\
\vdots & & \vdots & \vdots \\
\gamma(p-1) & \cdots & \gamma(0) & 0 \\
0 & \cdots & 0 & 1 / 2 \sigma^{2}
\end{array}\right)
$$

For the sequence of processes $\left\{\Psi_{\lambda}^{m}(\theta)\right\}$, we have that

$$
\Psi_{\lambda}^{m}(\theta) \rightarrow I(\theta)^{1 / 2} W(\lambda)
$$

where " $\rightarrow$ " denotes weak convergence and where $W$ is a $(p+1)$-dimensional Wiener process with independent components $W_{i}$, see Theorem VIII.3.33 in Jacod and Shiryaev (1987).

Let $\hat{\theta}$ be the conditional maximum likelihood [ML] estimator, to be found by solving the likelihood equations

$$
\sum_{t=1}^{m} \rho_{t}(\theta)=0
$$

where $\rho_{t}(\theta)$ is as defined in (7). If $\hat{\theta}=\left(\hat{\phi}^{\prime}, \hat{\sigma}^{2}\right)^{\prime}, \hat{\phi}$ is actually the least squares estimator, and $\hat{\sigma}^{2}=m^{-1} \sum_{t=1}^{m} \hat{\varepsilon}_{t}^{2}$, where $\hat{\varepsilon}_{t}=y_{t}-\hat{\phi}_{1} y_{t-1}-\cdots-\hat{\phi}_{p} y_{t-p}$.

The estimated score process is obtained by replacing the parameters in (9) by their estimates, resulting in

$$
\hat{\Psi}_{\lambda}^{m}=m^{-1 / 2} \sum_{t=1}^{[m \lambda]} \rho_{t}(\hat{\theta}) .
$$

The sequence of processes $\left\{\hat{\Psi}_{\lambda}^{m}\right\}$ converges weakly to a Gaussian process, that is,

$$
\hat{\Psi}_{\lambda}^{m} \rightarrow I(\theta)^{1 / 2} W^{0}(\lambda)
$$

where $W^{0}(\lambda)=W(\lambda)-\lambda W(1)$, which follows directly from the multivariate functional limit theorem, see also Chu et al. (1996, p. 1054).

\subsection{An autoregression of order 2}

For illustration, we consider the case with $p=2$ and compute the elements of

$$
I(\theta)^{-1 / 2} \Psi_{\lambda}^{m}(\theta)
$$

Let $n$ be the integer part of $m \lambda$, that is, $n=[m \lambda]$. The two first components of this vector, which we denote by $S^{\phi}$, concern the two autoregressive parameters. We have

$$
S_{n}^{\phi}=\sigma\left(\begin{array}{ll}
\gamma(0) & \gamma(1) \\
\gamma(1) & \gamma(0)
\end{array}\right)^{-1 / 2} \sum_{t=1}^{n}\left[\begin{array}{l}
y_{t-1} \varepsilon_{t} / \sigma^{2} \\
y_{t-2} \varepsilon_{t} / \sigma^{2}
\end{array}\right] .
$$


For this case, $\gamma(0)$ and $\gamma(1)$ are given by

$$
\left\{\begin{array}{l}
\gamma(0)=\frac{1-\phi_{2}}{\left(1+\phi_{2}\right)\left[\left(1-\phi_{2}\right)^{2}-\phi_{1}^{2}\right]} \sigma^{2} \\
\gamma(1)=\frac{\phi_{1}}{\left(1+\phi_{2}\right)\left[\left(1-\phi_{2}\right)^{2}-\phi_{1}^{2}\right]} \sigma^{2}
\end{array}\right.
$$

see Fuller (1996, p 55). Upon using this, we can show that

$$
\sigma\left(\begin{array}{ll}
\gamma(0) & \gamma(1) \\
\gamma(1) & \gamma(0)
\end{array}\right)^{-1 / 2}=\sqrt{1+\phi_{2}} P\left(\begin{array}{cc}
\lambda_{2}^{1 / 2} & 0 \\
0 & \lambda_{1}^{1 / 2}
\end{array}\right) P^{\prime}
$$

where $\lambda_{1}$ and $\lambda_{2}$ are the eigenvalues of the matrix $\left(\begin{array}{cc}1-\phi_{2} & \phi_{1} \\ \phi_{1} & 1-\phi_{2}\end{array}\right)$, that is, $\lambda_{1}=1-\phi_{2}-\phi_{1}, \lambda_{2}=1-\phi_{2}+\phi_{1}$ and where $P=1 / \sqrt{2}\left(\begin{array}{cc}1 & 1 \\ -1 & 1\end{array}\right)$. Using
these results gives

$$
S_{n}^{\phi}=\sqrt{1+\phi_{2}} P\left(\begin{array}{cc}
\lambda_{2}^{1 / 2} & 0 \\
0 & \lambda_{1}^{1 / 2}
\end{array}\right) P^{\prime} \sum_{t=1}^{n}\left[\begin{array}{l}
y_{t-1} \varepsilon_{t} / \sigma^{2} \\
y_{t-2} \varepsilon_{t} / \sigma^{2}
\end{array}\right]
$$

Finally, consider the variance of the error process, for which we have

$$
S_{n}^{\sigma^{2}}=\frac{1}{\sqrt{2}} \sum_{t=1}^{n}\left(\frac{\varepsilon_{t}^{2}}{\sigma^{2}}-1\right)
$$

From (12), it follows that

$$
m^{-1 / 2}\left(\begin{array}{c}
S_{[m \lambda]}^{\phi} \\
S_{[m \lambda]}^{\sigma^{2}}
\end{array}\right) \rightarrow W(\lambda),
$$

and if we replace the parameters by their respective estimates, we have

$$
m^{-1 / 2} \hat{S}_{[m \lambda]} \rightarrow W^{0}(\lambda)
$$

where

$$
\hat{S}_{n}=\left(\begin{array}{c}
S_{n}^{\hat{\phi}} \\
S_{n}^{\hat{\sigma}^{2}}
\end{array}\right)
$$




\section{The monitoring procedure}

In this section we incorporate the test statistic based on the score process into a monitoring procedure. Additionally, we evaluate its empirical performance under the null hypothesis in a limited simulation experiment.

\subsection{The procedure}

We aim to apply the score-based test in the following context. A set of $m$ observations has been collected and the parameters are estimated from this, say, historical data set. Next, we want to check if this estimated model fits equally well to newly arriving observations. We want our test to be valid as long as we get new data, that means, we do not want the size of the procedure to increase to unity as we repeat the test. The monitoring procedure for structural change described in Chu, Stinchcombe and White (1996) is most appropriate for these purposes. To save space, we will only highlight a few features of their approach.

If the sequence of processes $X_{\lambda}^{m} \equiv\left\{m^{-1 / 2} S_{[m \lambda]} \lambda \in[0, \infty]\right\}$ converges weakly to a Wiener process, then

$$
\begin{array}{r}
\lim _{m \rightarrow \infty} P\left\{\left|S_{n}\right| \geq \sqrt{m} g(n / m) \text { for some } n \geq m\right\} \\
=P\{|W(\lambda)| \geq g(\lambda) \text { for some } \lambda \geq 1\},
\end{array}
$$

where $g$ is a suitably chosen function. This expression also holds for multivariate processes. When one takes the function $g(\lambda)=\left[\lambda\left(a^{2}+\log \lambda\right)\right]^{1 / 2}$, where $a$ is a positive constant, the probability that a Wiener process crosses this function is

$$
\alpha(a)=2[1-\Phi(a)+a \phi(a)]
$$

where $\Phi$ and $\phi$ are the cdf and pdf of a standard normal distribution, see Chu et al. (1996). Consequently, the probability that at least one of the components of the $k$-dimensional process $W$ crosses the function $g$ is

$$
\tilde{\alpha}(a)=1-(1-2[1-\Phi(a)+a \phi(a)])^{k} .
$$

For further results, we refer to Corollary 3.6 in Chu et al. (1996, p. 1055).

For $k=3$, which corresponds to the $\operatorname{AR}(2)$ model, where we aim to evaluate the properties of the two AR parameters and the variance, taking $a^{2}=$ 10.1984 gives $\tilde{\alpha}(a)=0.05$ and $a^{2}=8.625$ gives $\tilde{\alpha}(a)=0.10$. Hence, the null hypothesis will be rejected when at least one of the components of the process $\hat{S}_{n}$ crosses a convenient boundary function. Hence our method can best be used as a general misspecification test.

\subsection{Some Monte Carlo evidence}

We evaluate the above monitoring procedure in a limited Monte Carlo simulation experiment. We generate $m+q$ observations from an $\operatorname{AR}(2)$ model with mean zero, unit variance for $\varepsilon_{t}$ and AR parameters $(1.2,-0.7)$ and $(0.3,-0.1)$, 
Table 1. Empirical size of the monitoring procedure: zero-mean $\operatorname{AR}(2)$ model with $\phi_{1}=1.2$ and $\phi_{2}=-0.7$

Asymptotic size 0.05

\begin{tabular}{|l|llllll|}
\hline$m$ & 50 & 75 & 100 & 200 & 500 & 800 \\
\hline \hline$q$ & & & & & & \\
\hline $2 m$ & 19.9 & 17.7 & 16.4 & 13.4 & 12.6 & 11.0 \\
$4 m$ & 22.1 & 19.3 & 17.7 & 14.2 & 13.4 & 11.4 \\
$6 m$ & 22.8 & 19.9 & 18.1 & 14.6 & 13.6 & 11.6 \\
$9 m$ & 23.5 & 20.5 & 18.4 & 14.9 & 13.7 & 11.7 \\
$19 m$ & 24.3 & 20.9 & 18.8 & 15.3 & 13.9 & 11.8 \\
\hline
\end{tabular}

Asymptotic size 0.10

\begin{tabular}{|r|rrrrrr|}
\hline \multicolumn{1}{|l|}{$q$} & \multicolumn{7}{|l|}{ A } & & & & & \\
\hline $2 m$ & 23.4 & 21.5 & 20.1 & 17.6 & 16.6 & 15.3 \\
$4 m$ & 25.9 & 23.4 & 21.8 & 18.9 & 17.9 & 16.0 \\
$6 m$ & 26.8 & 24.1 & 22.3 & 19.5 & 18.2 & 16.3 \\
$9 m$ & 27.5 & 24.8 & 22.9 & 19.9 & 18.4 & 16.5 \\
$19 m$ & 28.2 & 25.5 & 23.4 & 20.1 & 18.6 & 16.7 \\
\hline
\end{tabular}

Table 2. Empirical size of the monitoring procedure: zero-mean $\operatorname{AR}(2)$ model with $\phi_{1}=0.3$ and $\phi_{2}=-0.1$

Asymptotic size 0.05

\begin{tabular}{|l|llllll|}
\hline$m$ & 50 & 75 & 100 & 200 & 500 & 800 \\
\hline \hline$q$ & & & & & & \\
\hline $2 m$ & 14.4 & 14.0 & 12.0 & 9.2 & 8.4 & 7.6 \\
$4 m$ & 15.6 & 15.0 & 12.9 & 9.9 & 9.2 & 8.1 \\
$6 m$ & 16.1 & 15.4 & 13.1 & 10.2 & 9.4 & 8.3 \\
$9 m$ & 16.6 & 15.8 & 13.3 & 10.4 & 9.5 & 8.4 \\
$19 m$ & 16.9 & 16.0 & 13.7 & 10.5 & 9.6 & 8.5 \\
\hline
\end{tabular}

Asymptotic size 0.10

\begin{tabular}{|r|rrrrrr|}
\hline \multicolumn{1}{|l|}{$q$} & & & & & & \\
\hline $2 m$ & 18.4 & 17.7 & 15.6 & 13.4 & 12.3 & 11.8 \\
$4 m$ & 19.9 & 19.0 & 16.8 & 14.4 & 13.1 & 12.6 \\
$6 m$ & 20.5 & 19.6 & 17.1 & 14.5 & 13.3 & 13.0 \\
$9 m$ & 21.0 & 20.1 & 17.7 & 14.7 & 13.5 & 13.2 \\
$19 m$ & 21.5 & 20.4 & 18.2 & 15.0 & 13.8 & 13.4 \\
\hline
\end{tabular}

respectively. Next we apply the monitoring procedure and compute the frequency of rejection of the null hypothesis at nominal size 0.05 and 0.10 . The results are summarized in Tables 1 and 2.

The empirical size values in these tables indicate that the test is slightly oversized. Interestingly, it seems that the value of $q$ does not matter much, ex- 
Table 3. Finite sample power for an $\mathrm{AR}(2)$ model when only the AR parameters change: $\phi_{1}=(1.2,-0.4)^{\prime}$ to $\phi_{2}=(1.2,-0.7)^{\prime}$

Asymptotic size 0.05

\begin{tabular}{|l|cccccc|}
\hline$m$ & 50 & 75 & 100 & 200 & 500 & 800 \\
\hline \hline$q$ & & & & & & \\
\hline $2 m$ & 59.4 & 72.4 & 82.0 & 98.9 & 100 & 100 \\
$4 m$ & 70.8 & 83.5 & 92.1 & 99.9 & 100 & 100 \\
$6 m$ & 74.8 & 87.2 & 94.6 & 99.9 & 100 & 100 \\
$9 m$ & 78.0 & 89.8 & 96.2 & 100 & 100 & 100 \\
$19 m$ & 81.1 & 92.0 & 97.6 & 100 & 100 & 100 \\
\hline
\end{tabular}

Asymptotic size 0.10

\begin{tabular}{|c|cccccc|}
\hline \multicolumn{1}{l|}{$q$} & & & & & & \\
\hline $2 m$ & 64.7 & 77.3 & 86.0 & 98.0 & 100 & 100 \\
$4 m$ & 75.8 & 87.8 & 94.6 & 99.7 & 100 & 100 \\
$6 m$ & 79.4 & 90.1 & 96.8 & 99.9 & 100 & 100 \\
$9 m$ & 82.4 & 92.9 & 97.9 & 100 & 100 & 100 \\
$19 m$ & 85.4 & 94.8 & 99.0 & 100 & 100 & 100 \\
\hline
\end{tabular}

cept for very small samples. For practical work, we would recommend to set $m$ at a reasonably large value.

To study the finite sample power of the monitoring procedure, we simulate changes in the AR parameters $\phi$ and the variance of the innovation process $\sigma^{2}$. Three kinds of change are considered. First only the AR parameters change, that is, at time $t=[m * 1.1], \phi$ shifts from $(1.2,-0.4)$ to $(1.2,-0.7)$, while $\sigma^{2}$ remains unchanged $\left(\sigma^{2}=1\right)$. The relevant results are gathered in Table 3 . Next only $\sigma^{2}$ is subject to change. At time $t=[m * 1.1], \sigma^{2}$ decreases from 1 to 0.5 , while $\phi$ is constant at $(1.2,-0.7)$. The results are in Table 4 . Finally we consider the case where all parameters change. At time $t=[m * 1.1], \sigma^{2}$ decreases from 1 to 0.5 and the AR parameters shift from $(1.2,-0.4)$ to $(1.2,-0.7)$. The results are summarized in Table 5.

The simulation results in these table are easy to summarize. For $m \geq 200$ and $q \geq 4 m$, the empirical power is close or equal to 1 . The method is also quite powerful in small samples when only the AR parameters change.

\section{Monthly US industrial production}

In this section we apply our monitoring procedure to US industrial production. In the empirical business cycle literature, there is a substantial interest in the possible stabilization of the US economy throughout the twentieth century, see for example, Watson (1994) and the references cited therein. If an autoregression with complex roots would describe the growth rates in industrial production, stabilization would mean that AR parameters change such that the implied cycle length gets shorter. However, one may also define stability in terms of a reduced variance. Hence, significant stabilization would emerge as timevarying parameters in an autoregression. 
Table 4. Finite sample power for an $\operatorname{AR}(2)$ model when only the variance of the error process changes: $\sigma_{1}^{2}=1$ to $\sigma_{2}^{2}=0.5$. The parameter $\phi$ remains constant, $\phi=(1.2,-0.7)^{\prime}$

Asymptotic size 0.05

\begin{tabular}{|l|rccccc|}
\hline$m$ & \multicolumn{1}{|l|}{50} & 75 & 100 & 200 & 500 & 800 \\
\hline \hline$q$ & & & & & & \\
\hline $2 m$ & 9.6 & 13.5 & 28.2 & 90.4 & 100 & 100 \\
$4 m$ & 10.9 & 25.4 & 51.0 & 98.2 & 100 & 100 \\
$6 m$ & 12.2 & 31.9 & 61.1 & 99.1 & 100 & 100 \\
$9 m$ & 13.7 & 37.7 & 67.7 & 99.6 & 100 & 100 \\
$19 m$ & 15.8 & 43.5 & 74.0 & 99.7 & 100 & 100 \\
\hline
\end{tabular}

Asymptotic size 0.10

\begin{tabular}{|c|c|c|c|c|c|c|}
\hline$q$ & & & & & & \\
\hline $2 m$ & 12.6 & 25.6 & 46.8 & 95.9 & 100 & 100 \\
\hline $4 m$ & 18.1 & 43.7 & 69.9 & 99.3 & 100 & 100 \\
\hline $6 m$ & 21.8 & 51.8 & 77.3 & 99.7 & 100 & 100 \\
\hline $9 m$ & 25.3 & 57.7 & 81.5 & 99.8 & 100 & 100 \\
\hline $19 m$ & 29.2 & 63.6 & 85.9 & 99.9 & 100 & 100 \\
\hline
\end{tabular}

Table 5. Finite sample power for an $\operatorname{AR}(2)$ model when both the variance of the error process and the autoregressive parameters change: $\sigma_{1}^{2}=1$ to $\sigma_{2}^{2}=0.5$ and $\phi_{1}=(1.2,-0.4)^{\prime}$ to $\phi_{2}=(1.2,-0.7)^{\prime}$

Asymptotic size 0.05

\begin{tabular}{|l|llllll|}
\hline$m$ & 50 & 75 & 100 & 200 & 500 & 800 \\
\hline \hline$q$ & & & & & & \\
\hline $2 m$ & 22.3 & 26.9 & 34.2 & 75.1 & 100 & 100 \\
$4 m$ & 26.7 & 34.0 & 45.2 & 92.0 & 100 & 100 \\
$6 m$ & 28.4 & 37.3 & 51.4 & 95.8 & 100 & 100 \\
$9 m$ & 30.0 & 40.1 & 55.6 & 97.2 & 100 & 100 \\
$19 m$ & 32.0 & 43.5 & 61.8 & 98.3 & 100 & 100 \\
\hline
\end{tabular}

Asymptotic size 0.10

\begin{tabular}{|r|rrrrrr|}
\hline \multicolumn{1}{l|}{$q$} & & & & & & \\
\hline $2 m$ & 26.2 & 34.6 & 45.3 & 87.8 & 100 & 100 \\
$4 m$ & 31.9 & 45.5 & 60.6 & 97.5 & 100 & 100 \\
$6 m$ & 34.8 & 49.8 & 67.3 & 98.5 & 100 & 100 \\
$9 m$ & 37.0 & 53.5 & 72.4 & 99.0 & 100 & 100 \\
$19 m$ & 39.7 & 58.3 & 77.9 & 99.5 & 100 & 100 \\
\hline
\end{tabular}




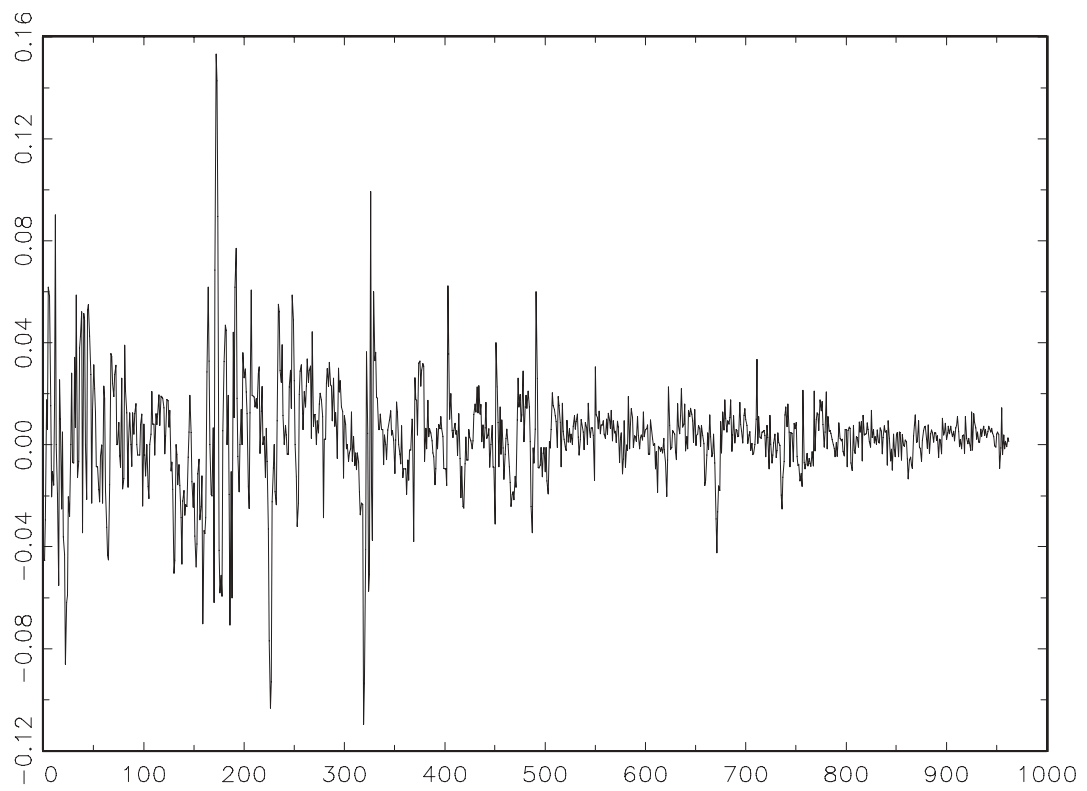

Fig. 1. First differences of the (log) monthly US industrial production index (1919.01-1999.05)

We consider the monthly growth rate in (seasonally adjusted) US industrial production index, observed for 1919.01 to 1999.05 for which we find an $\operatorname{AR}(2)$ model to describe the data reasonably well, as indicated by diagnostic tests on the estimated residuals. The data appear in Figure 1. Clearly, there is visual evidence of changing unconditional variance in this series, but whether this is due to changes in the AR parameters and/or the variance of the error process is not immediately obvious. Therefore, we use our monitoring procedure and we display the results, when we compare pre-war data $(m=336)$ with post-war data $(q \simeq 2 m)$, in Figure 2. The empirical results lead to a clear-cut conclusion, that is, the parameters in this autoregression are not constant over time.

In a next step we calculate a Wald test for the constancy of the AR parameters and a Goldfeld-Quandt test for the constancy of the variance, where we set the break date equal to 1946.01 (which corresponds with $m=336$ ). The Wald test obtains a value 13.45 of while the second test obtains a value of 7.98. As both statistics indicate rejection of the null hypothesis, we conclude that the $\mathrm{AR}$ and the variance parameters both changed over time. The AR parameters changed from $(0.59,-0.10)$ to $(0.38,0.13)$, and the innovation variance from $7.510^{-4}$ to $9.410^{-5}$.

It may also be of interest to restrict attention to post-war data. When we compare the first 10 years of post-war growth rate $(m=120)$ with more recent observations $(q \simeq 4 m)$, again upon considering an $\operatorname{AR}(2)$ model, we find again evidence of time-varying parameters, see Figure 3. The corresponding Wald test and Goldfeld-Quandt test for constant AR parameters now take values of 0.65 and 2.6. Here we find that the AR parameters seem constant, and that only the innovation variance has changed in the post war period. This variance changed from $1.810^{-4}$ to $7.210^{-5}$. 

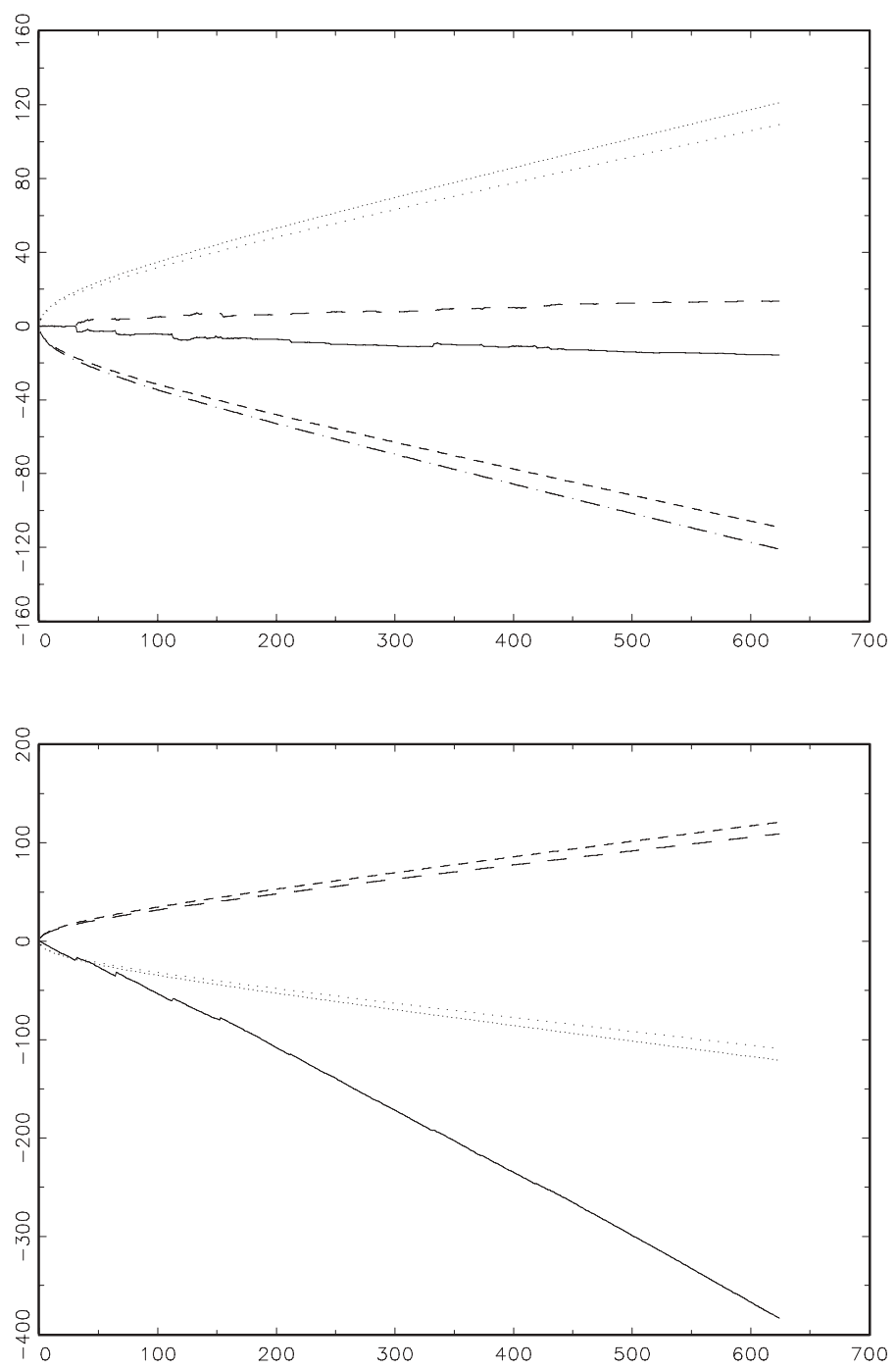

Fig. 2. Comparing pre-war and post-war data $(m=336)$. The top panel concerns $S^{\hat{\phi}}$ and the bottom panel concerns $S^{\hat{\sigma}^{2}}$.

In sum, we find strong evidence in favor of a reduction of the innovation variance in an autoregression for US industrial production.

\section{Conclusion}

In this paper we adapted the monitoring procedure of Chu et al. (1996) to investigate structural breaks in the parameters of an autoregression. The procedure was evaluated by Monte Carlo simulations, and these suggested that it has reasonable size and good power properties. We applied our method to monthly US industrial production data, for which we found evidence of stabilization, 

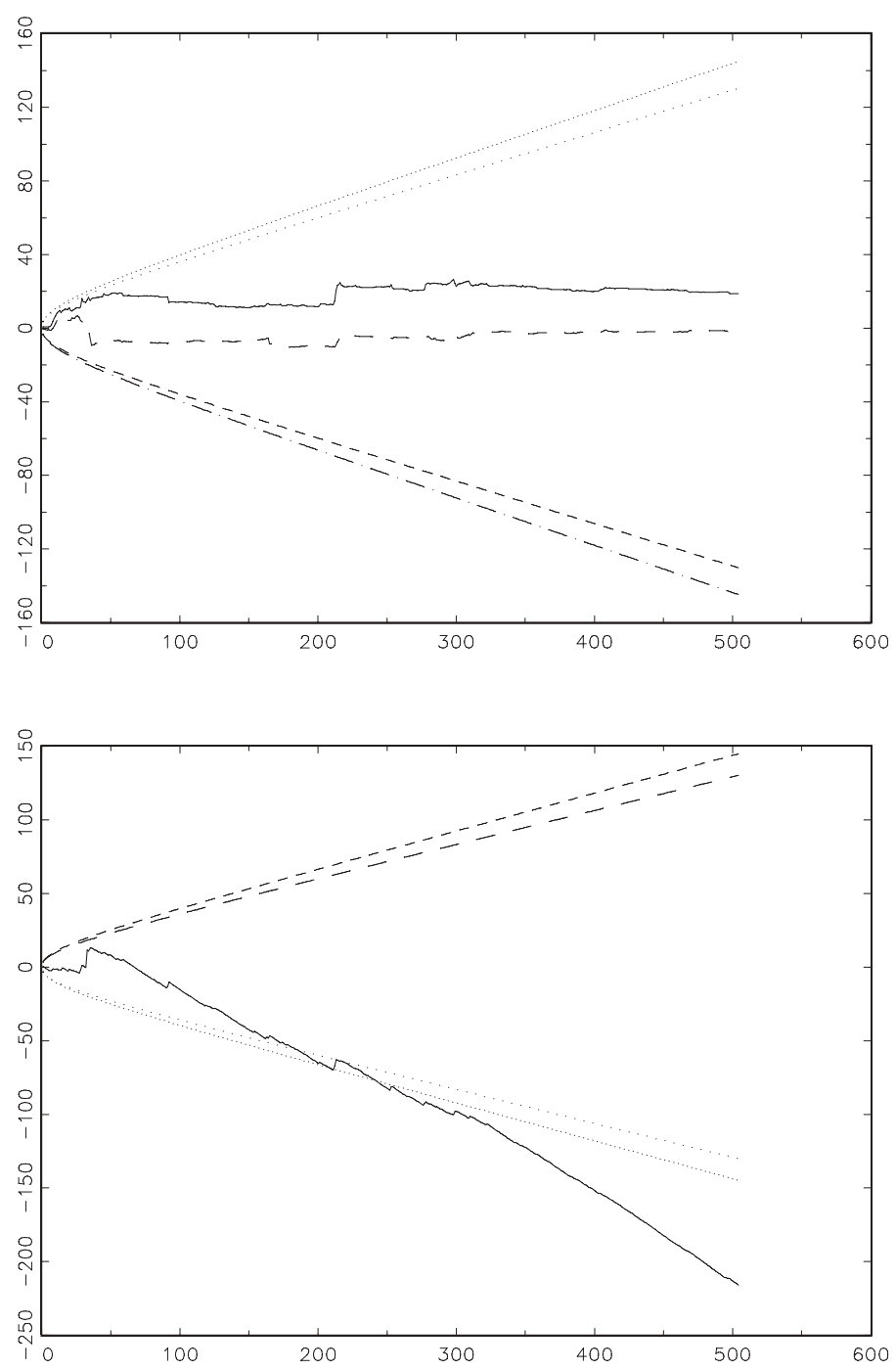

Fig. 3. Is there sructural a break in post-war data? $(m=120)$ The top panel concerns $S^{\hat{\phi}}$ and the bottom panel concerns $S^{\hat{\sigma}^{2}}$.

where this increased stability appeared to be mainly due to a reduction in the variance of the innovations.

\section{References}

Chu CSJ, Stinchcombe M, White H (1996) Monitoring structural change. Econometrica 64:10451065

Fuller WA (1996) Introduction to statistical time series, 2nd edition. New York: Wiley Jacod J, Shiryaev AN (1987) Limit theorems for stochastic processes. Berlin: Springer Verlag Watson MW (1994) Business-cycle duration and post war stabilization for the US economy. American Economic Review 84:24-46 